ARTICLE

\title{
MLLT1 YEATS domain mutations in clinically distinctive Favourable Histology Wilms tumours
}

\author{
Elizabeth J. Perlman', Samantha Gadd', Stefan T. Arold², Anand Radhakrishnan², Daniela S. Gerhard ${ }^{3}$, \\ Lawrence Jennings ${ }^{1}$, Vicki Huff ${ }^{4}$, Jaime M. Guidry Auvil ${ }^{3}$, Tanja M. Davidsen ${ }^{3}$, Jeffrey S. Dome ${ }^{5}$, \\ Daoud Meerzaman ${ }^{6}$, Chih Hao Hsu ${ }^{6}, \mathrm{Cu}_{\text {Nguyen }}{ }^{6}$, James Anderson ${ }^{7}$, Yussanne Ma ${ }^{8}$, Andrew J. Mungall ${ }^{8}$, \\ Richard A. Moore ${ }^{8}$, Marco A. Marra ${ }^{8}$, Charles G. Mullighan ${ }^{9}$, Jing Ma ${ }^{9}$, David A. Wheeler ${ }^{10}$, \\ Oliver A. Hampton ${ }^{10}$, Julie M. Gastier-Foster ${ }^{11,12}$, Nicole Ross ${ }^{11} \&$ Malcolm A. Smith ${ }^{13}$
}

Wilms tumour is an embryonal tumour of childhood that closely resembles the developing kidney. Genomic changes responsible for the development of the majority of Wilms tumours remain largely unknown. Here we identify recurrent mutations within Wilms tumours that involve the highly conserved YEATS domain of MLLT1 (ENL), a gene known to be involved in transcriptional elongation during early development. The mutant MLLT1 protein shows altered binding to acetylated histone tails. Moreover, MLLT1-mutant tumours show an increase in MYC gene expression and HOX dysregulation. Patients with MLLT1-mutant tumours present at a younger age and have a high prevalence of precursor intralobar nephrogenic rests. These data support a model whereby activating MLLT1 mutations early in renal development result in the development of Wilms tumour.

\footnotetext{
${ }^{1}$ Department of Pathology, Ann \& Robert H. Lurie Children's Hospital of Chicago, Northwestern University's Feinberg School of Medicine, 225 E. Chicago Ave, Chicago, Illinosis 60611, USA. ${ }^{2}$ King Abdullah University of Science and Technology, Department of Biochemistry and Molecular Biology, Division of Biological and Environmental Sciences and Engineering, Computational Bioscience Research Center, Thuwal 23955, Saudi Arabia. ${ }^{3}$ Office of Cancer Genomics, National Cancer Institute, 31 Center Drive, Bethesda, Maryland 20892, USA. ${ }^{4}$ Department of Genetics, The University of Texas MD Anderson Cancer Center, 1515 Holcombe Blvd., Houston, Texas 77030, USA. ${ }^{5}$ Department of Pediatrics, Division of Pediatric Hematology/Oncology, Children's National Medical Center, 111 Michigan Avenue, NW, Washington DC 20010, USA. ${ }^{6}$ Center for Biomedical Informatics and Information Technology, National Cancer Institute, National Institutes of Health, 9609 Medical Center Drive, Bethesda, Maryland 20892, USA. ${ }^{7}$ Frontier Science and Technology Research Foundation, 505 S. Rosa Rd \#100, Madison, Wisconsin 53719, USA. ${ }^{8}$ Canada's Michael Smith Genome Sciences Centre, British Columbia Cancer Agency, Vancouver, British Columbia, Canada V5Z 4S6. ${ }^{9}$ Department of Pathology, St Jude Children's Research Hospital, 262 Danny Thomas Place, Mail Stop 342, Memphis, Tennessee 38105 , USA. ${ }^{10}$ Department of Pathology and Laboratory Medicine, Nationwide Children's Hospital, Ohio State University College of Medicine, Columbus, Ohio 43205, USA. ${ }^{11}$ Department of Molecular and Human Genetics, Baylor College of Medicine, Houston, Texas 77030, USA. ${ }^{12}$ Departments of Pathology and Pediatrics, Ohio State University College of Medicine, 700 Children's Drive, Columbus, Ohio 43205, USA. ${ }^{13}$ Cancer Therapy Evaluation Program, National Cancer Institute, 9609 Medical Center Drive, RM 5-W414, MSC 9737, Bethesda, Maryland 20892, USA. Correspondence and requests for materials should be addressed to E.J.P. (email: Eperlman@luriechildrens.org).
} 
W ilms tumour (WT), the most common embryonal neoplasm of the childhood kidney, has long intrigued investigators by its histologic similarity to the developing kidney ${ }^{1}$. WTs are also noteworthy for their associated precursor lesions known as perilobar and intralobar nephrogenic rests (PLNR, ILNR) ${ }^{2}$. Favourable histology Wilms tumours (FHWT) represent $95 \%$ of WT; the remaining $5 \%$ develop an additional clonal event (most commonly TP53 mutation) resulting in unfavourable histology characterized as anaplasia (nuclear hyperchromasia and pleomorphism with polyploid mitoses $)^{3,4}$. Molecular alterations long known to contribute to WT development include mutations and/or deletions involving WT1, WTX, CTNNB1 and loss of imprinting or loss of heterozygosity $(\mathrm{LOH})$ at $11 \mathrm{p} 15$ (reviewed in ref. 5). However, the genomic changes responsible for the development of most WTs remain unknown. The National Cancer Institute's 'Therapeutically Applicable Research to Generate Effective Treatments' (TARGET) initiative seeks to identify therapeutic targets for high-risk paediatric tumours through comprehensive characterization and integrative analyses (http://ocg.cancer.gov/programs/target). The ongoing High Risk TARGET Wilms Tumours include two groups: FHWT that subsequently relapsed and anaplastic WT.

We report in-frame insertion/deletion MLLT1 mutations in FHWT that are absent in other TARGET paediatric tumour types. These MLLT1 mutations were associated with changes in binding to $\mathrm{H} 3 \mathrm{~K} 9 \mathrm{ac}$, evidence of $\mathrm{HOX}$ and $M Y C$ (MYC-C) expression dysregulation, and were often accompanied by CTNNB1 mutations and evidence of Wnt pathway activation. Further, patients with MLLT1 mutations presented at a younger age and were associated with ILNR rather than PLNR. We conclude that activating MLLT1 mutations accompanied by Wnt activation early in renal development result in the development of Wilms tumour.

\section{Results}

Identification and characterization of MLLT1 variants. As we recently reported, analysis of 77 FHWT by whole genome sequencing (WGS, 58 patients) or whole exomic sequencing (WES,19 patients) identified 825 high-quality somatic, non- synonymous variants, with an average of 11 candidate mutations/ case (range 2-42) (ref. 6). Six variants (all identified by WGS) involved MLLT1, a gene not previously reported to be involved in WT. Each mutation was verified by Sanger sequencing of PCR products spanning the variants (Fig. 1). Other verified mutations identified include somatic single nucleotide variants (SNVs) or small deletions in WT1 (three patients, 4.5\%), CTNNB1 (five patients, 6.5\%), WTX (also known as FAM123B or AMER1, five patients, 6.5\%), DROSHA (seven patients, 10\%), DGCR8 (three patients, $4.5 \%$ ), and SIX1 or SIX2 (eight tumours, $10 \%)^{6}$. Two tumours with MLLT1 mutations also had CTNNB1 mutations; no MLLT1-mutant tumours had accompanying WT1, WTX, DROSHA, DGCR8, SIX1, or SIX2 mutations (Supplementary Table 1). These represent all genes with verified variants in at least four patients with FHWT ( $>5 \%$ ); the full TARGET highrisk Wilms tumour data, including 125 tumours (both favourable histology and anaplastic tumours) and including verified variants present at lower frequencies, will be reported in full when these data become available.

Four cases had the identical nine nucleotide in-frame insertion (AACCACCTG) at MLLT1 position c.343_351 (p.117_ 118insNHL) of the transcript NM_005934.3. Two mutations were nearby in-frame deletions, one with deletion of six nucleotides at position c.335_340 (p.112_114PPV $>$ L) and one with a seven-nucleotide deletion and a one-nucleotide insertion at position c.333_339 (p.111_113NPP > K) (Supplementary Table 1). The MLLT1 mutations were present in $49-73 \%$ of the sequencing reads and reverse transcription PCR (RT-PCR) analysis found expression of both wild-type and mutant alleles. Similar MLLT1 insertion/deletion variants were not identified in other TARGET tumours (neuroblastoma, osteosarcoma, acute lymphocytic and myelogenous leukaemia, unpublished), nor have they been reported in dbSNP 134 or 135 (ref. 7), COSMIC v69 (ref. 8), or in the 1000 Genomes Pilot Projects 1, 2, and 3 (ref. 9). The PROVEAN algorithm ${ }^{10}$ predicted these MLLT1 mutations to be deleterious (scores $<-10)$. PCR amplification followed by sequencing identified an additional MLLT1 p.117_118insNHL mutation in 1/ 19 tumours originally characterized by WES, for a total of 7 MLLT1 mutations in 77 discovery set tumours (11\%) (Supplementary Table 1). No germline MLLT1 mutations were identified.
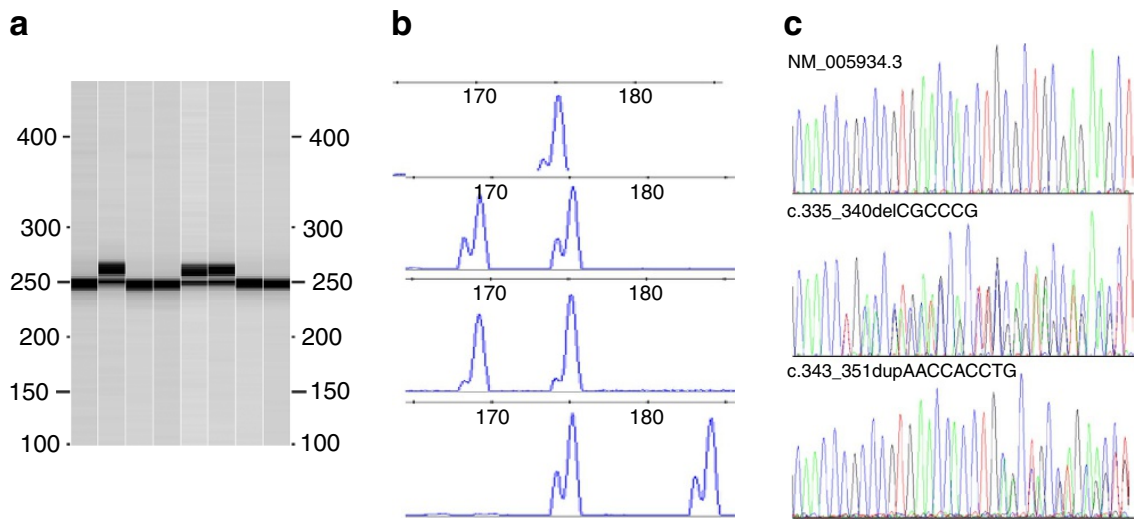

Figure 1 | MLLT1 variant verification. (a) Base-pairs (242) of genomic DNA were amplified and the product was detected by capillary electrophoresis. Eight representative samples with three containing additional larger bands identifying insertion mutations (lanes 2, 5 and 6) are shown. Samples with novel bands were confirmed by Sanger sequencing. (b) The relative expression of the mutant and wild-type alleles was determined using RT-PCR and primers designed to amplify exons 4 and 5 of the MLLT1 gene (NM_ 005934) with detection by capillary electrophoresis. The normal allele is represented by a peak at $\sim 175 \mathrm{bp}$ (top panel). Two samples show expression of a shorter allele corresponding to deletion mutations (second and third panels) and one sample shows expression of a longer allele (bottom panel) corresponding to an insertion mutation. It should be noted that the mutant alleles are expressed at approximately the same level as the normal alleles. (c) Samples with abnormal bands identified following PCR amplification were confirmed by Sanger dideoxy sequencing. Shown are three samples including the reference sequence on top (NM_005934.3), a 6-nucleotide deletion in the middle (c.335_340delCGCCCG) and a 9-nucleotide duplication on the bottom (c.343_351dupAACCACCTG). The base-pair positions have been aligned to show that these affect the same domain. 
Copy number and gene expression analysis. Copy number loss at 19 p13 (the chromosomal location of MLLT1) was not identified in any of the 77 FHWT; five non-mutant tumours had gain of 19 p13 due to gain of all or the majority of chromosome 19. Segmental copy number loss was identified for WT1 (14 tumours, three showing loss of small segments containing all or part of WT1 and the remainder loss of the majority or all of chromosome 11) and WTX (19 tumours, 14 showing loss of small segments containing all or part of WTX and the remainder loss of the majority or all of the X chromosome) (Fig. 2, Supplementary Table 1). Gene expression analysis was available for 75 of the 77 tumours (one MLLT1-mutant tumour and one MLLT1 wild-type tumour did not pass quality control). As we recently reported within the same 75 tumours, unsupervized analysis using nonnegative matrix factorization (NMF) resulted in $k=6$ clusters having the highest cophenetic correlation (0.95) after $k=2$ (ref. 6). We are now able to identify five of six MLLT1-mutant tumours within NMF cluster 3 (Fig. 2). Comparison of global gene expression of MLLT1-mutant tumours with 69 MLLT1 wild-type tumours yielded 96 genes significantly differentially expressed (two-class unpaired significance analysis of microarrays $q<0.05$, Bonferroni-corrected Student's $t$-test $p$ value $<0.05$, fold change $>2$, Supplementary Table 2). Noteworthy was the significantly increased expression of HOXA13, HOXB6, HOXB7, MYC, PRAC, IRX3 and PITX2, and decreased expression of HOXD10 and HOXD11 (Fig. 2). Of particular interest was the high expression of HOXA13 (fold change 128), a gene not expressed in the remaining FHWT nor in the cap mesenchyme of the developing kidney ${ }^{11}$. The expression of HOXA13 mRNA has been shown to require the expression of the immediately adjacent lncRNA HOTTIP (refs 12,13). Six MLLT1-mutant tumours and six randomly selected $M L L T 1$-wild-type FHWTs were analysed by quantitative reverse transcription PCR, which confirmed increased expression of both HOXA13 and HOTTIP in MLLT1mutant tumours (Fig. 3a). Increased expression of other lncRNA/ target pairs, including $P R A C$ and its adjacent lncRNA $H O X B-A S$ (PRAC2, NCRNA00253) (ref. 14) and IRX5 and its adjacent lncRNA CRNDE (ref. 15) was also observed (Supplementary Table 2, Fig. 2). The increased expression of these critical lncRNAs detected with array analysis is particularly noteworthy because lncRNAs are not usually expressed at levels detectable using these methods.

To validate the functional impact of the MLLT1 mutations on gene expression, we transfected the MLLT1 p.117_118insNHL and p.112_114PPV $>\mathrm{K}$ mutant constructs alone or at a 1:1 ratio with the wild-type $M L L T 1$ construct into the embryonic kidney cell line HEK293. Both HOXA13 and HOTTIP were significantly upregulated in the cells transfected with either of the mutant MLLT1 genes alone and in the cells transfected with the 1:1 ratio of mutant:wild-type MLLT1 (Fig. 3b) compared with the vector transfected control cells and the cells transfected with wildtype alone. To confirm that the MLLT1 mutations did not alter protein stability, cell lysates from the transfected cells were evaluated by western analysis at 24 48, and $72 \mathrm{~h}$ (Fig. 3c and Supplementary Fig. 2), revealing no significant differences in mutant protein expression levels.

MLLT1 mutation clinical and genetic correlations. The TARGET FHWT cases were selected as high risk on the basis of their clinical recurrence. To determine the frequency of MLLT1 mutations overall in FHWT, we screened a validation set of 475 FHWT by PCR amplification and sequencing. MLLT1 insertion or deletion mutations were found in 19/475 tumours (4\%). The

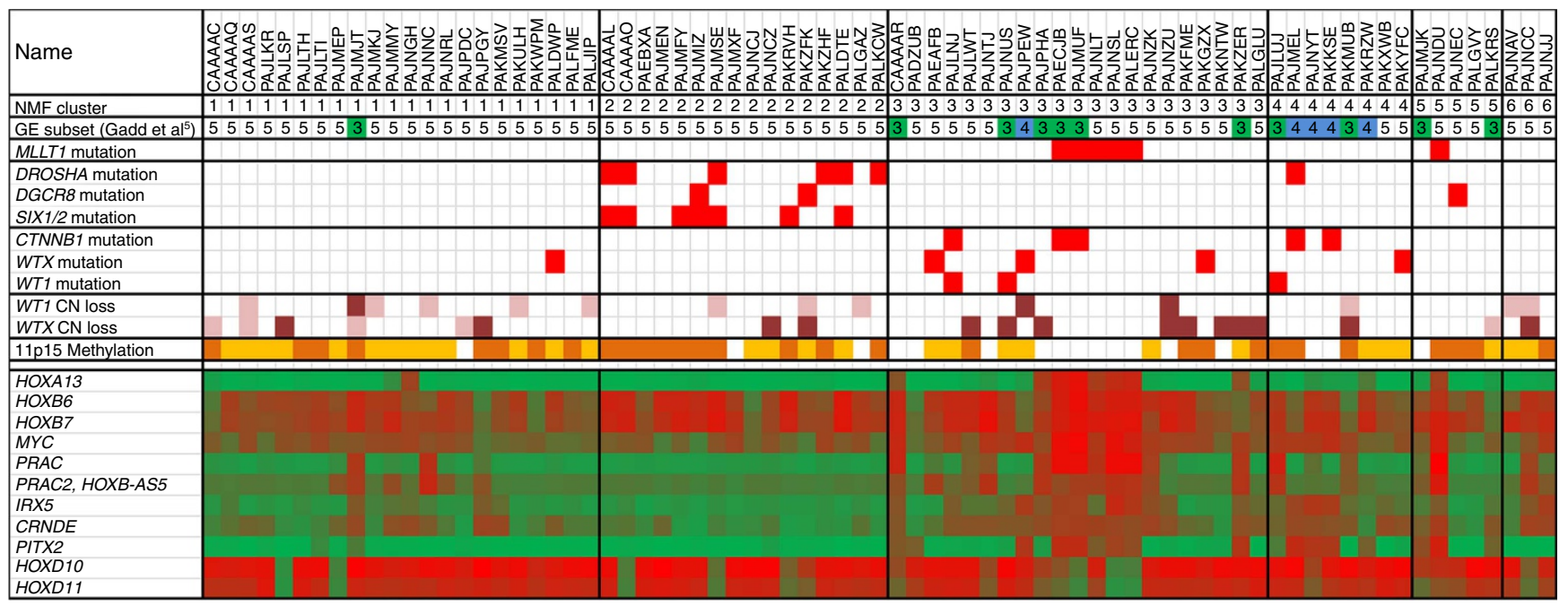

Log2 expression value

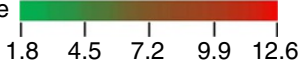

WT1 and WTX copy number loss:

Small segmental loss Entire chromosome arm loss
GE subset:

Subset 3

Subset 4

Subset 5
11 15 Methylation:

Loss of Heterozygosity s of imprinting Retention of imprinting

Figure 2 | Unsupervized analysis of gene expression data. Non-negative Matrix Factorization (NMF) analysis of 75 FHWT resulted in 6 clusters with the highest cophenetic correlation (0.95) after $k=2$. Five of six MLLT1 mutant tumours with available gene expression data occurred in NMF cluster 3, and two were accompanied by CTNNB1 mutations. This cluster also contained four tumours with mutation or small segment deletion of WT1, all of which also had either a mutation of CTNNB1 or small segment deletion or mutation of WTX. This cluster also contained a substantial number of tumours with retention of imprinting of 11p15 (including all MLLT1-mutant tumours). The sequencing and copy number data is provided in Supplementary Table 1. The predicted membership in gene expression subsets previously reported ${ }^{5}$ is provided. (Subsets 1 and 2 were low risk tumours and are therefore not represented in TARGET, Subsets 3 and 4 are highlighted in green and blue, respectively, and Subset 5 in white). Illustrated at the bottom are the expression patterns of genes of interest that were highly significantly differentially expressed between MLLT1-mutant and wild-type tumours. 
a

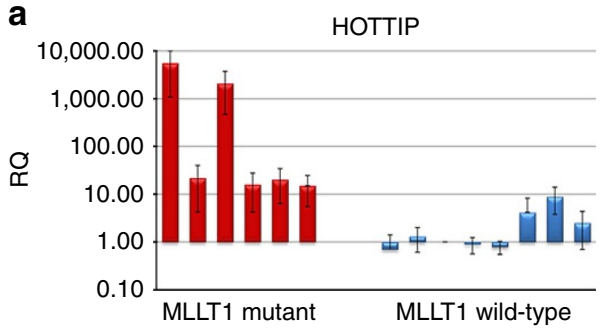

b

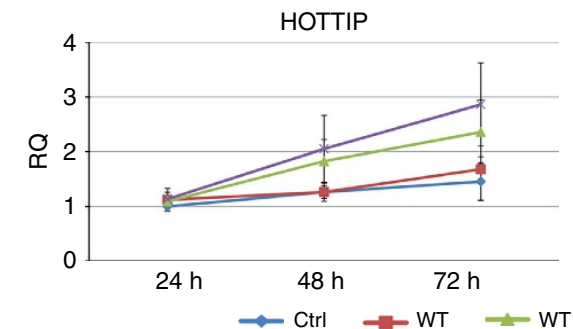

HOTTIP

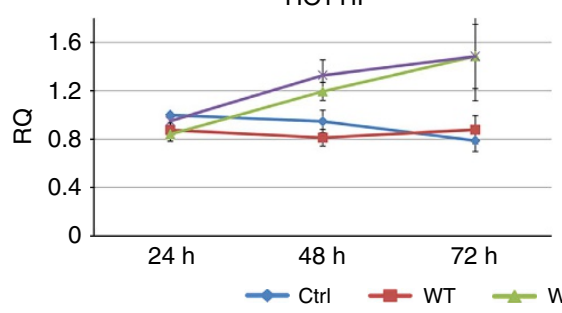

c

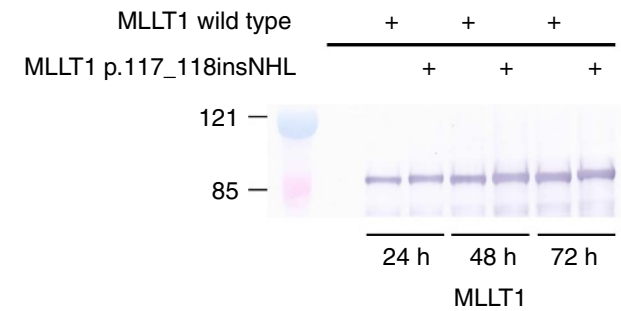

C

MLLT1
HOXA13

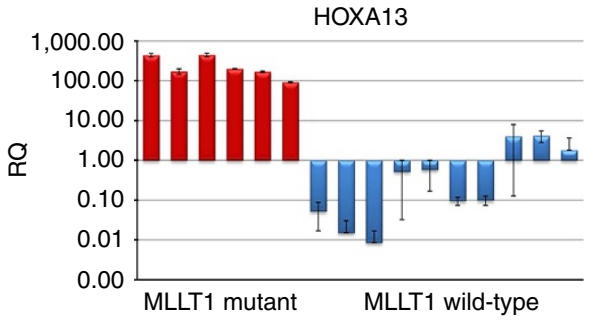

HOXA13

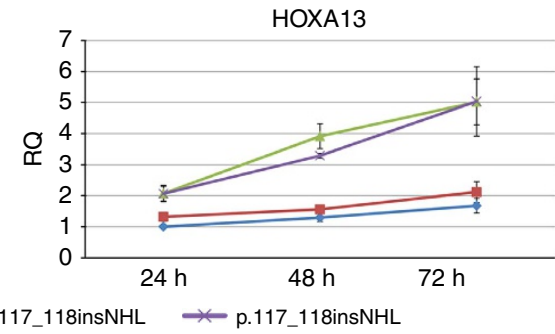

HOXA13

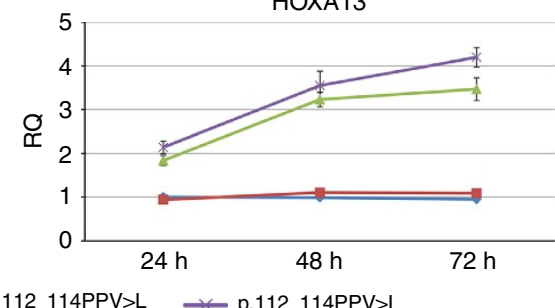

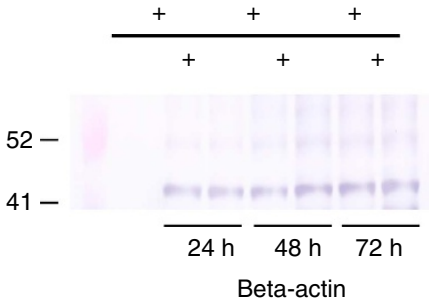

Figure 3 | Quantitative RT-PCR for HOTTIP and HOXA13. (a) FHWT samples with MLLT1 mutations ( $n=6$; red) and randomly selected MLLT1-wild-type FHWT ( $n=10$; blue) were evaluated for HOTTIP and HOXA13 expression levels by RT-PCR normalized to endogenous GAPDH levels. A FHWT lacking MLLT1 mutation was used as the reference sample for calculating the relative quantitation (RQ) value. Samples were run in duplicate and the error bars represent the s.e.m. of the RQ values. The data are presented in log scale. Note that for tumours that lack expression a bar is not visible. (b) HEK293 cells transfected with empty vector (CtI), wild-type MLLT1 (WT), a 1:1 ratio of wild-type MLLT1 and either p.117_118insNHL (upper panels) or p.112_114PPV>K (lower panels), or only mutant p117_118insNHL or p.112_114PPV $>\mathrm{K}$ is shown. RNA was isolated 24, 48 and $72 \mathrm{~h}$ after transfection, and the expression levels of HOXA13 and HOTTIP were normalized to endogenous GAPDH levels. The ddCt value for the control sample at $24 \mathrm{~h}$ was used as the reference sample for calculating the RQ value in each sample for each gene. Three independent experiments were performed for each transfection and the error bars represent the s.e.m. of the RQ values. (c) HEK293 cells transfected with wild-type MLLT1 or mutant MLLT1 (p117_118insNHL). Lysates were collected at 24, 48 , and $72 \mathrm{~h}$ for western blotting with a MLLT1 (left) or beta-actin (antibody). The mutant protein was not significantly differentially expressed compared with the wild-type protein at any of the time points evaluated. Results for p.112_114PPV > K likewise demonstrated no significant difference in levels of protein expression.

p.117_118insNHL mutation was identified in 13/19 tumours. Three identical new variants were also identified as p.117_118insHHL. The remaining three were deletion mutations at p.111-112 (Table 1). MLLT1 mutations were observed in 6\% (9/139) of FHWT that recurred, compared with 3\% (10/336) of FHWT that did not recur. Although this suggests an association between MLLT1 mutation and relapse, this difference does not meet statistical significance. There was also no detectable correlation between the specific MLLT1 mutation and relapse.

The clinical, pathologic and molecular details of the 19 FHWT carrying somatic MLLT1 mutations compared with the entire group of 475 FHWT are provided in Table 1 . The median age at diagnosis of the patients with and without MLLT1 mutations was
12 and 44 months, respectively, ( $P<0.0001$, Wilcoxon test). There was also a significant correlation between MLLT1 mutation status and nephrogenic rest status. Of MLLT1-wild-type tumours, 23 and 21\% were associated with ILNRs and PLNRs, respectively; in contrast, $10 / 19(53 \%, P=0.011)$ MLLT1-mutant tumours arose in ILNRs, and $0 / 19(P=0.019)$ arose in PLNRs (Fisher's exact test). Four MLLT1-mutant tumours arose in kidneys with multiple ILNRs, a feature thus far predominantly recognized in patients with syndromes associated with germline WT1 mutations. Of note, in 5/7 MLLT1-mutant tumours in the discovery set, the comparator constitutional sample was adjacent normal kidney and lacked the mutation. A source of constitutional DNA was available for the four cases in the validation set that had 


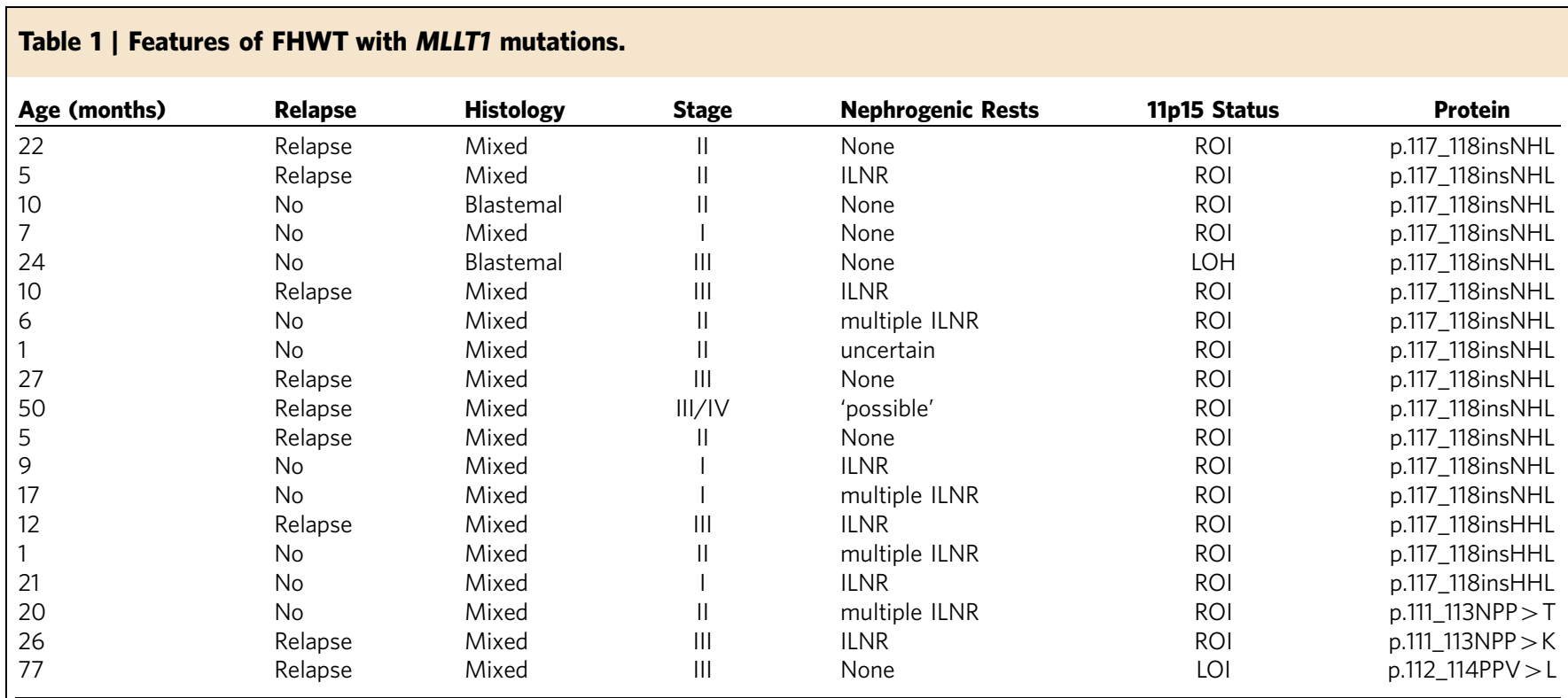

ILNR, Intralobar nephrogenic rest; LOH, loss of heterozygosity; LOI, Loss of imprinting; ROI, retention of normal imprinting.

multiple ILNRs (two from peripheral blood and two from adjacent normal kidney) and these samples likewise lacked MLLT1 variants by PCR amplification and sequencing. Sufficient DNA was available on 16/19 MLLT1-mutant tumours to perform mutation analysis for WT1, CTNNB1, WTX, DROSHA, DGCR8, and SIX1/2 and this revealed five CTNNB1 mutations, one WTX mutation, and no WT1 DROSHA, DGCR8, or SIX1/2 mutations.

A different prevalence of other genetic markers was identified in MLLT1-mutant tumours. Overall, $81 \%$ of all FHWT have either LOH or loss of imprinting at ICR1 on 11p15 as determined by methylation analysis ${ }^{5}$; in comparison, only $2 / 18$ (11\%) FHWT with MLLT1 mutations with available material demonstrated such changes (Table 1). LOH of $1 \mathrm{p}$ and $16 \mathrm{q}$ are known to be associated with relapse in patients with $\mathrm{FHWT}^{16}$. Information regarding $1 \mathrm{p}$ and $16 \mathrm{q} \mathrm{LOH}$ analysis performed prospectively during the protocol was available for 11/19 MLLT1-mutant tumours, and neither was found. Similarly, 1q gain is present in 57 and $24 \%$ of patients with FHWT that relapsed and do not relapse, respectively ${ }^{17}$. 1q gain was identified in $1 / 15$ MLLT1mutant FHWT (see methods). Thus, although MLLT1 mutation was often associated with poor outcome, this association was independent of other prognostic markers.

MLLT1 protein evaluation. The MLLT1 locus encodes a 559aa protein from 12 exons and is one of over 100 proteins that contain a YEATS domain. The domain name is derived from the five proteins first discovered in either Saccharomyces cereviseiae or humans, including Yaf9 (YEATS4, GAS41), ENL (MLLT1, YEATS1), AF9 (MLLT3, YEATS3), Taf14 and Sas5. In humans, MLLT1 and AF9 are the most studied and are highly homologous ${ }^{18}$. Using a variety of structural and functional analyses, Li et al. recently demonstrated the AF9 YEATS domain to (1) span N-terminal residues $1-138$, (2) form an immunoglobulin fold family $\beta$-sandwich with eight antiparallel $\beta$ strands separated by nine loops capped by $\alpha$ helices and (3) function as a reader protein, recognizing and binding $\mathrm{H} 3 \mathrm{~K} 9 \mathrm{ac}$ (ref. 19). Given their reported homology ${ }^{19}$, we used the crystallographic three-dimensional structure of the AF9 YEATS domain as a template to construct a computational model for MLLT1 YEATS domain. The MLLT1 YEATS sequence is $82 \%$ identical to AF9 and has no amino acid insertions or deletions
(Fig. 1a). Therefore the two structures are expected to be extremely similar, resulting in a highly reliable computational three-dimensional model for the MLLT1 YEATS domain, with a QMEAN (qualitative model energy analysis) $Z$ score of +0.49 . (The QMEAN $Z$ score ranges from -4 (worse) to +4 (best) with the average QMEAN $Z$ score for high-resolution X-ray structures being 0) (ref. 20). The AF9 amino acids demonstrated by Li et al. to bind H3K9ac are located in loops 1, 4 and 6; these amino acids are identical in MLLT1 (Fig. 4a,b). Important residue differences between MLLT1 and AF9 involve positions 107 and 111 within loop 8 (L8) and these are the same amino acids demonstrated by Li et al. to be responsible for binding of AF9 to the -5 position with respect to the H3K9ac motif (H3K4). It is striking that all MLLT1 mutations specifically affect L8; mutations involving positions 111 and 112 directly impact L8, and those involving position 117 insert NHL or the similar NHH into the $\beta 8$ strand (both sequences are equally capable of binding with the adjacent $\beta 1$ strand in the place of the native NHL), pushing the native NHL out of $\beta 8$ and into L8 (Fig. 4a-c). Our computational homology modelling indicates that all the mutations could be accommodated within the overall YEATS fold without significantly distorting it (Fig. 4d). Indeed, when we produced wild-type and mutant MLLT1 recombinant polypeptides, the mutations did not significantly affect the protein's heat stability measured by differential scanning fluorimetry (melting temperature with $95 \%$ confidence interval for the mean of three experiments: wild-type $T \mathrm{~m}=47.3 \pm 0.3^{\circ} \mathrm{C}$, p.117_118insNHL $\quad T \mathrm{~m}=47.1 \pm 0.1{ }^{\circ} \mathrm{C}, \quad$ p.111_113NPP $>\mathrm{K}$ $\mathrm{Tm}=47.3 \pm 0.3^{\circ} \mathrm{C}, \quad$ p. $\left.112 \_114 \mathrm{PPV}>\mathrm{L} \quad \mathrm{Tm}=46.7 \pm 0.3^{\circ} \mathrm{C}\right)$, arguing against loss of function through simple YEATS domain misfolding. (We did not test p.117_118insHHL, because this mutant was predicted to show the same structure and hence thermostability and binding characteristics as p.117_118insNHL).

The AF9 YEATS domain has its highest affinity to H3K9ac, with somewhat lower affinity binding to H3K27 (ref. 19). We therefore tested the ability of recombinant wild-type and mutant MLLT1 YEATS domain polypeptides to bind acetylated and nonacetylated $\mathrm{H} 3 \mathrm{~K} 9$ and $\mathrm{H} 3 \mathrm{~K} 27$ using isothermal titration calorimetry (ITC). Wild-type MLLT1 bound to H3K9ac with a dissociation constant $K_{\mathrm{d}}$ of $60 \mu \mathrm{M}$, but failed to bind to non-acetylated H3K9 (Fig. 4e and Supplementary Fig. 1). The p.117_118insNHL mutation abolished the interaction with 
a

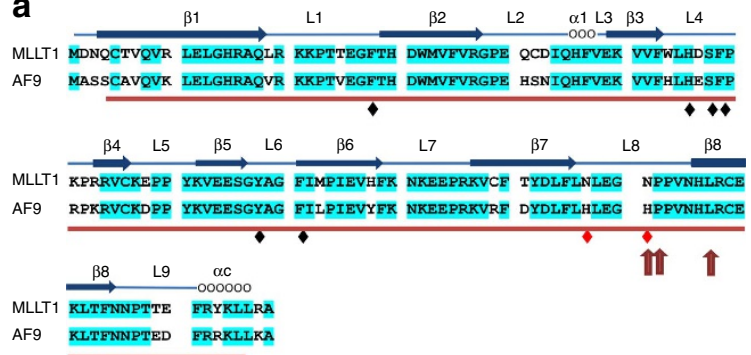

b
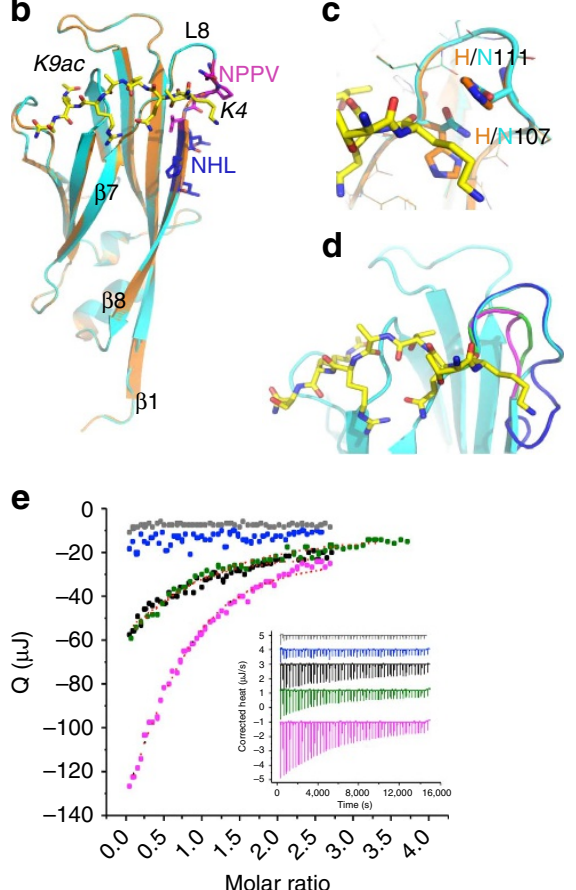

Figure 4 | MLLT1 computational sequence and structure analysis. (a) The sequences of the MLLT1 and AF9 YEATS domains are provided. Residues forming the YEATS domain are underlined in red. Identical residues are highlighted in blue. The secondary structural elements, including the $\beta$ strands and intervening loops described for AF9 (ref. 19) are provided. The recognition sites for $\mathrm{H} 3 \mathrm{~K} 9 \mathrm{ac}$ by AF9 are indicated with a black diamond; residue differences at positions 107 and 111 that are also recognition sites for H3K4 by AF9 are indicated with a red diamond ${ }^{19}$. Arrows indicate the MLLT1 mutations identified in the current study. (b) Homology model of the MLLT1 YEATS domain (cyan), superimposed on AF9 YEATS (orange) bound to H3K9ac (yellow stick model; PDB 4TMP). The motifs in which the indels occur are shown in blue and magenta letters. The $\beta 1, \beta 7, \beta 8$ strands and $L 8$ loop are designated (see text). (c) Amino acid changes between AF9 and MLLT1 in the L8 loop affect the recognition of the N-terminal region of acetylated lysine motifs. Residue differences $(\mathrm{H}>\mathrm{N})$ between AF9 and MLLT1 at positions 107 and 111 are highlighted in the superimposition of the AF9 crystal structure (PDB 4TMP; orange) with our homology model of MLLT1 YEATS (magenta). (d) The MLLT1 mutations are predicted to specifically affect the L8 loop. Computational homology models are coloured cyan (wild-type MLLT1), magenta ( $p .111 \_113 N P P>K$ ), green (p.112_114PPV $>$ L) and blue (p.117_118insNHL). p.117_118insHHL is predicted to produce the same conformation and phenotype as p.117_118insNHL, because N/H117 are solvent exposed at the back of $\beta 8$. (e) Effect of MLLT1 mutations on binding H3K9ac, measured by ITC, performed in triplicate. Shown are integrated heats resulting from binding of H3K9ac to MLLT1 wild-type (black), p.111_113NPP > K (magenta), p.112_114PPV $>$ L (green) and p.117_118insNHL (blue). Heats resulting from peptide being titrated into buffer are in grey. Inlay: ITC data adjusted to baseline and offset for better visibility.
H3K9ac, whereas p.112_114PPV $>\mathrm{L}$ weakened it $\left(K_{\mathrm{d}}=107 \mu \mathrm{M}\right)$. The p.111_113NPP $>\mathrm{K}$ mutation did not affect the binding affinity with $\mathrm{H} 3 \mathrm{~K} 9 \mathrm{ac}\left(K_{\mathrm{d}}=57 \mu \mathrm{M}\right)$, but altered the changes in entropy and enthalpy upon association, suggesting that the atomic interactions and dynamics differ from wildtype. With regard to binding to $\mathrm{H} 3 \mathrm{~K} 27 \mathrm{ac}$, wild-type MLLT1 showed substantially reduced affinity compared with H3K9ac $\left(K_{\mathrm{d}}=140\right.$ $\mu \mathrm{M}$,) and did not bind non-acetylated H3K27. The p.111_113NPP $>\mathrm{K}$ mutant polypeptide bound H3K27ac with an even lower affinity $(330 \mu \mathrm{M})$ (Supplementary Fig. 1). The p.112_114PPV $>$ L and p117_118insNHL mutants showed no detectable binding to H3K27ac under the conditions used. Thus, the novel MLLT1 mutations we describe have a clear effect on the capacity of MLLT1 YEATS to interact with $\mathrm{H} 3$ tail peptides. Compared with MLLT1, the AF9 YEATS domain bound H3K9ac and $\mathrm{H} 3 \mathrm{~K} 27$ with a higher affinity $\left(K_{\mathrm{d}}\right.$ of 3.7 and $7.0 \mu \mathrm{M}$, respectively $)^{19}$. This suggests the possibility that MLLT1 may require additional or different contacts for high-affinity binding than the ones tested.

\section{Discussion}

MLLT1 is a critical component of the super-elongation complex (SEC) that regulates mRNA elongation by RNA polymerase II (RNAPII) during transcription, and hence regulates RNA expression ${ }^{21-23}$. Perturbation of transcriptional elongation has been shown to result in abnormal development and neoplastic transformation (reviewed in ref. 24). The SEC contains a number of proteins, including AFF4, MLLT1 and PTFEb, among others. Mechanistically, the C-terminal region of MLLT1 (or the homologous AF9) binds to the scaffolding protein AFF4, which then binds the positive transcription elongation factor $b$ (PTEFb) and ELL2 through separate domains. The N-terminal YEATS domain binds the polymerase-associated factor complex (PAFc), which in turn associates with RNAPII and the chromatin template. PTEFb functions to phosphorylate the carboxy-terminal domain of RNAPII to facilitate transcriptional elongation ${ }^{25}$. MLLT1 and AF9 therefore bridge PTEFb (C terminus) with PAFC ( $\mathrm{N}$ terminus) and thereby with RNAPII, enabling transcriptional elongation. MLLT1 and AF9 compete with each other for binding AFF4 at their C terminus and exist in separate SEC complexes ${ }^{23}$; however, the functional differences between MLLT1- and AF9- bearing SEC complexes are not clear. Support for a critical role for MLLT1 within cellular biochemistry is provided by genetic ablation experiments in which MLLT1-deficient embryos die extremely early in utero, even before implantation ${ }^{26}$.

The MLLT1 and AF9 genes were originally recognized through their participation in chromosomal translocations that fuse their C-terminal domains (eliminating their YEATS domain) in-frame with the $\mathrm{N}$ terminus of MLL (eliminating the histone $\mathrm{H} 3 \mathrm{~K} 4$ methyltransferase activity but retaining the chromatin template binding function) within acute leukaemia ${ }^{27,28}$. These translocations result in bridging of PTEFb with RNAII similar to that described above, yet with important differences. The $\mathrm{N}$ terminus of MLL (which itself is able to bind PAFc and to the chromatin template of MLL-specific targets) fuses with MLLT1 (or AF9), thereby again bridging RNAPII with SEC and enabling the transcription of MLL target genes such as MEIS and HOXA9 (refs 21-23,29). It has long been speculated that the YEATS domain likewise functions by binding the chromatin template ${ }^{30,31}$; if this is the case, the specific genetic targets of MLLT1 likely differ from those of MLL.

In addition to AFF4 of the SEC, the C terminus of both MLLT1 and AF9 also bind to DOT1L ${ }^{19,32-34}$, which is the sole enzyme responsible for $\mathrm{H} 3 \mathrm{~K} 79$ methylation ${ }^{35,36}$. DOT1L was originally thought to be part of the SEC complex, however, evidence now 
suggests that the interaction between MLLT1/AF9 and AFF4 competes with the binding of MLLT1/AF9 with DOT1L (refs 22,23). Li et al. recently demonstrated that the AF9 $\mathrm{N}$-terminal YEATS domain specifically binds H3K9ac, bridging the chromatin template with DOT1L, resulting in $\mathrm{H} 3 \mathrm{~K} 79$ trimethylation, and hence transcriptional activation of associated genes ${ }^{19}$. They thereby establish that the AF9 YEATS domain serves as an acetyl-lysine reader protein and suggest that it functions similarly with respect to SEC (ref. 19). The $>80 \%$ homology between AF9 and MLLT1 YEATS domain supports a similar function for MLLT1. MLLT1 has been previously demonstrated to bind to histones 1 and 3 , however the preferred moiety for binding has not yet been identified ${ }^{30}$.

In the current study, we identified recurrent MLLT1 mutations that all target L8 of the YEATS domain. We demonstrated that wild-type MLLT1 indeed binds H3K9ac and that the MLLT1 mutations result in proteins with altered binding to $\mathrm{H} 3 \mathrm{~K} 9 \mathrm{ac}$. Therefore, our binding assays confirm that L8 plays an important role in modulating the affinity and specificity of the YEATS domain, and document a functional impact of the recurrent MLLT1 mutations. However, even wild-type MLLT1 binds to H3K9ac with a much lower affinity than does AF9. Therefore, it remains possible, if not likely, that $\mathrm{H} 3 \mathrm{~K} 9 \mathrm{ac}$ may not be the histone acetyl-lysine that MLLT1 binds to with the highest affinity. The underlying complexity of the functional impact of MLLT1 mutation is also suggested by the fact that reduced binding of AF9 with $\mathrm{H} 3 \mathrm{~K} 9 \mathrm{ac}$ resulted in decreased gene expression ${ }^{19}$; the increased expression of critical genes that we report and the presumed gain of function resulting from MLLT1 mutation are therefore difficult to explain. One of many possibilities is that decreased binding of mutant-MLLT1-bearing SEC may result in increased binding of AF9-bearing-SEC (which has a higher H3K9ac binding affinity than MLLT1). Further functional studies are needed to fully explore MLLT1 histone tail binding specificity. Moreover, the alteration in histone binding may not fully account for the functional impact of these mutations.

Other than histones, the only other protein known to bind the $\mathrm{N}$ terminus of MLLT1 is PAF1, a member of the PAF multi-protein complex that binds to the SEC and is also involved in cell cycle regulation, miRNA processing, $\mathrm{H} 3 \mathrm{~K} 4$ methylation and H3K79 methylation ${ }^{37,38}$. The dysregulation of several HOX genes and the upregulation of MYC (MYC-C) in MLLT1-mutant FHWT provide indirect support for an altered interaction between PAFc and the SEC in MLLT1-mutant tumours, as these genes are all recognized to be regulated through transcriptional elongation via the SEC (refs 23,39-41). The positive effect of the MLLT1 mutations on MYC expression may contribute to transformation through activation. The PAF complex is composed of four proteins, PAF1, CTR9, CDC73 and LEO1. Of considerable interest is the recent report of germline inactivating mutations within the CTR9 gene in three of 35 Wilms tumour families ${ }^{42,43}$, and the report of the development of Wilms tumours in patients with germline inactivating CDC73 mutations (hyperthyroidism-jaw tumour syndrome, OMIM 145001) (refs 44,45). These were coupled with somatic mutations of the remaining alleles, consistent with tumour suppressor function. Both CTR9 and CDC73 play a negative role in transcriptional elongation ${ }^{45}$, therefore inactivating mutations in these genes and activating mutations of MLLT1 may similarly result in transcriptional activation. We did not find somatic mutations in any of the genes in the PAFc in these 77 patients. Functional studies will be of considerable interest to define the precise changes in binding between mutant versus wild-type MLLT1 and components of the PAFc in addition to histones.

The absence of reports of these novel MLLT1 mutations in other tumours or tissues suggests the possibility that their impact may be restricted to a specific cellular context. Further, the observation that these mutations were identified in very young patients whose tumours arose within ILNR, precursor lesions thought to develop quite early in renal development ${ }^{2,46}$, provides evidence that this particular cellular context may be limited to the early embryonic kidney. Many WT with WT1 mutations also present at a young age, occur within ILNRs and develop as early as the intermediate mesoderm ${ }^{5}$. Constitutional WT1 mutations often result in multiple ILNRs and WTs ${ }^{46}$. These WT1-mutant tumours of infancy require Wnt pathway activating mutations (for example, CTNNB1, WTX) for transformation ${ }^{5,47}$. It is therefore noteworthy that $37 \%$ of the evaluable MLLT1-mutant tumours (which lacked WT1 mutations and deletions) also had CTNNB1 or WTX mutations, and MLLT1-mutant tumours all show a striking increase in expression of PITX2 (Fig. 2), a gene encoding a homeobox protein whose expression follows canonical Wnt pathway activation and results in increased proliferation during early development ${ }^{48,49}$. The identification of multiple ILNRs in some patients with MLLT1-mutant WTs is intriguing, and raises questions regarding the possibility of germline mutations. We were unable to identify any evidence of MLLT1 germline mutations, even in the MLLT1-mutant tumours with multiple ILNRs. Nor were we able to identify MLLT1 mutations in normal kidney adjacent to MLLT1-mutant tumours. Last, one of the authors (VH) provided DNA samples from 48 families with familial WT. These were tested for MLLT1 mutations and all were negative (data not shown). In summary, we did not find evidence to support the existence of germline MLLT1 mutations in FHWT. It may be speculated that in patients with ILNRs and whose tumours carried MLLT1 mutations, these mutations occurred in an undifferentiated cell early in kidney development. The resulting clone of undifferentiated cells could remain localized, or as the early kidney grows they could separate into multiple aggregates, resulting in the subsequent development of single or multiple ILNRs. WT may then develop within an ILNR, possibly following mutations resulting in Wnt pathway activation. This model would predict that, unlike the adjacent normal kidney tissue we assessed for several patients with MLLT1-mutant tumours, ILNRs from these patients would carry the MLLT1 mutation. Unfortunately, ILNR material was unavailable to test this.

Evidence for HOX dysregulation mediated by MLLT1 mutation in FHWT is most abundantly seen in the striking and early overexpression of HOXA13. HOXA13 is not expressed in normal renal development, and the specific role of HOXA13 overexpression in WT development is not apparent. However, it is critical for the development of the distal limbs and the reproductive system; in fact, HOXA13 mutation is responsible for the rare handfoot-uterus syndrome (OMIM 140000). HOXA13 upregulation has also been shown to be a marker of poor outcome in a number of adult cancers (reviewed in ref. 50). Mechanistically, the association between MLLT1 mutation and HOXA13 overexpression seen in our mutant transfection analysis is supported by the accompanying increased expression of HOTTIP, a lncRNA that has been reported to result in transcriptional activation of HOXA13 (ref. 12). The aberrant expression of critical lncRNAs (and their targets) in MLLT1-mutant FHWT may simply reflect underlying general mechanisms affecting both lncRNAs and targets. However, one might speculate that MLLT1 may participate more broadly in the regulation of transcription mediated by lncRNAs, in keeping with a proposed model whereby lncRNAs act as scaffolds that brings regulatory protein complexes (such as SEC) together into larger functional units in order to coordinate cell-type-specific gene expression programmes ${ }^{51}$.

In summary, we report novel somatic mutations in MLLT1 and provide evidence that supports a role for MLLT1 mutation in the 
aetiology of a subset of FHWT. Our data indicates that understanding the function of these MLLT1 mutations could provide important insight into the function of the YEATS domain in other proteins, and into transcriptional regulation during early renal development as well as tumour development.

\section{Methods}

TARGET data management. The NCI TARGET initiative specifies and supports the long-term deposition and maintenance of the data files, methods and quality control steps involved in the comprehensive genomic analysis of TARGET samples. The sequencing BAM files from both WES and WGS are deposited in the Sequence Read Archive at the National Center for Biotechnology Information, and are accessible through the database of genotypes and phenotypes (dbGAP, http://www.ncbi.nlm.nih.gov/gap) under the accession number phs000471. Gene expression, chromosome segmental copy number and the clinical information are available through the TARGET data matrix (http://target.nci.nih.gov/dataMatrix/ TARGET_DataMatrix.html). These are annotated within MIAME compliant MAGE-TAB files fully describing the methods, the specimen processing details and the quality control parameters.

Specimens. Primary, pre-therapy tumour and paired blood or normal kidney samples from 77 patients registered on NWTS-5 with FHWT who subsequently relapsed were included in the discovery set. To determine the mutation frequency and to identify clinical associations, a validation set was identified comprised of a previously defined case-cohort of FHWTs likewise treated on NWTS-5 (including those in the discovery set) ${ }^{5}$. This approach allows for efficient evaluation of molecular markers in a tumour type characterized by a low rate of relapse. In brief, a cohort of all 1473 patients with FHWT enroled on NWTS-5 who were treated according to protocol and who had samples submitted to the NWTSG tumour bank in 2003 were identified. Following the methods of Prentice ${ }^{52}$, a random sample of $33 \%$ of the cohort was selected. To this group, 178 cases from the entire cohort who were known to have relapsed as of 2003 were added. This resulted in a total of 600 patients, $30 \%$ of whom relapsed. Of the 600 cases in the original casecohort, 475 continued to have available tumour DNA samples in 2012 and received adjuvant chemotherapy. (Patients registered on the very low risk protocol who did not receive adjuvant chemotherapy were removed as all patients with MLLT1 mutation received adjuvant chemotherapy). The validation set included tumours from these 475 patients, 139 of whom relapsed. The median time to relapse for FHWT is $<1$ year, and $<1 \%$ of patients with FHWT relapse beyond 5 years ${ }^{53}$ NWTS- 5 closed for accrual in 2002, therefore the clinical outcome data is fully mature. Written consent was obtained from the parents and the studies were performed with the approval of the Ann \& Robert H. Lurie Children's Hospital Institutional Review Board.

Sequencing. WGS was accomplished through the four adaptor library protocol ${ }^{54}$ and variants were identified through a pipeline that is described on the TARGET DataMatrix. The variants were filtered retaining all somatic, non-synonymous variants in exons with a somatic score $>-10$, somatic rank $\geqslant 0.1$, FET $P<0.05$. WES was performed on the Illumina HiSeq platform. Variant calling from the aligned BAM files was performed using both ATLAS and SAMtools and annotation and filtering was performed using the SACBE annotation pipeline ${ }^{55,56}$ as well as Bambino Version 1.05 (ref. 57). Pipelines for both WGS and WES identified single missense, insertion and deletion variants in exons.

MLLT1 variant verification. Primers were designed to amplify a 242 base-pair sequence of genomic DNA that included the sites for both the insertion and deletions identified. PCR amplification was performed using Platinum PCR SuperMix High Fidelity (Life Technologies Corporation, Carlsbad, CA). Cycling conditions were as follows: initial denaturation was performed at $96^{\circ} \mathrm{C}$ for $2 \mathrm{~min} ; 5$ cycles were performed at $96^{\circ} \mathrm{C}$ for $20 \mathrm{~s}, 60^{\circ} \mathrm{C}$ for $50 \mathrm{~s}$, and $72^{\circ} \mathrm{C}$ for $30 \mathrm{~s} ; 30$ cycles were performed at $94{ }^{\circ} \mathrm{C}$ for $22 \mathrm{~s}, 55^{\circ} \mathrm{C}$ for $50 \mathrm{~s}, 72{ }^{\circ} \mathrm{C}$ for $30 \mathrm{~s}$; a final extension was performed at $72{ }^{\circ} \mathrm{C}$ for $10 \mathrm{~min}$. The amplified product was detected using capillary electrophoresis (QIAxcel Advanced System, Qiagen, Valencia, CA). Positive samples were confirmed by Sanger sequencing using a Big Dye Terminator v1.1 cycle sequencing kit (Life Technologies Corporation, Carlsbad, CA) and M13 forward and M13 reverse primers as sequencing primers. The DNA sequence analysis was performed with 3130xl Genetic Analyzer Data Collection software v3.0 (Life Technologies Corporation, Carlsbad, CA).

Forward primer with M13F: $5^{\prime}$-TGTAAAACGACGGCCAGTGTGCCCTGA GAGAGAAGTGG-3'

Reverse primer with M13R: 5'-CAGGAAACAGCTATGACCTGAAGGTGA GCTTCTCG-3'

MLLT1 gene expression analysis. The relative expression of the mutant and wild-type alleles was determined using 2-step RT-PCR. Primers were designed to target exons 4 and 5 of the MLLT1 gene (NM_ 005934). Complementary DNA was synthesized using the SuperScript VILO cDNA kit from Invitrogen. The manufacture protocol was followed and $300 \mathrm{ng}$ of total RNA was used per reaction. Briefly, the complementary DNA (cDNA) synthesis reaction was incubated at $25^{\circ} \mathrm{C}$ for $10 \mathrm{~min}, 42^{\circ} \mathrm{C}$ for $120 \mathrm{~min}$ and $85^{\circ} \mathrm{C}$ for $5 \mathrm{~min}$. The PCR reaction mixture was assembled from a 2XddPCR Supermix for Probes (Bio-Rad, Cat\#1863010), with a final of concentration of $900 \mathrm{nM}$ for each primer and $3 \mathrm{ul}$ of $\mathrm{CDNA}$ in a final volume of $20 \mu \mathrm{l}$. Cycling conditions were as follows: denaturation was performed at $95^{\circ} \mathrm{C}$ for $10 \mathrm{~min}$; 40 cycles were performed at $94{ }^{\circ} \mathrm{C}$ for $30 \mathrm{~s}$ and $59^{\circ} \mathrm{C}$ for $50 \mathrm{~s}$; a final extension was performed at $98^{\circ} \mathrm{C}$ for $10 \mathrm{~min}$. The amplified product was detected using capillary electrophoresis (3130xl Genetic Analyzer, Life Technologies Corporation, Carlsbad, CA).

MLLT1-RT-PCR-fwd /56-5'-FAM/CTGTTCCTGAACCTGGAAGG-3'

MLLT1-RT-PCR-rev 5' ${ }^{\prime}$-TGGGTAACATGGGGTAGTCG-3'

Chromosome segment copy number analysis. DNA labelling, hybridization, and array scanning were performed for SNP 6.0 arrays (Affymetrix, Santa Clara, CA) according to the manufacturer's protocol and processed with the Affymetrix Genotyping Console 4.0 software to generate Birdseed .chp and .txt files using the Birdseed v2 algorithm with the default parameters., Model-based expression analysis was performed and imported into $\mathrm{R}$ for reference normalization to a diploid reference chromosome determined for each tumour, resulting in a normalized probe level summarization value and a genotype call for each individual probe ${ }^{58}$. Circular binary segmentation was performed in R using the DNAcopy BioConductor package. Default parameters included nperm $=10,000$ (the number of permutations used for Pvalue calculation), $\alpha=0.01$ (the significance level for the test to accept change points), and random seed $=12,345,678$ (the seed for the random number generator). This resulted in a segmented file for each tumour sample relative to the corresponding normal sample. Segments containing at least eight markers in which the $\log 2$ value was $\geq+0.5$ or $\leq-0.5$ were considered regions of gain or loss, respectively.

Gene expression analysis. RNA labelling, hybridization, and array scanning were performed according to the manufacturer's protocol for the Affymetrix U133 plus 2 arrays. Unsupervized analysis was performed using the Non-negative Matrix Factorization (NMF) Consensus Version 5 (ref. 59), applied to the level 3 data using the following parameters: $\mathrm{k}$ initial, 2; $\mathrm{k}$ final, 8; number of clusterings, 20; maximum number of iterations, 2,000; error function, divergence; random seed, 12,345,6789; stop convergence, 40; stop frequency, 10. Comparisons of gene expression between two groups of samples were performed using the Significance Analysis of Microarray Version 4.0 (ref. 60) with the following parameters: twoclass unpaired comparison using the T-statistic test, 200 permutations, and a fold change cutoff of $+/-2$.

Methylation analysis at 11p15. Analysis of the extent of methylation of two imprint control regions (ICR) on chromosome 11 p15 was performed taking advantage of specific restriction sites recognized by methylation-sensitive enzyme as previously described ${ }^{61}$. In brief, DNA was extracted using the Qiagen DNeasy Blood and Tissue Kit (Qiagen, Santa Clarita, CA) following the manufacturer's protocol. DNA was digested with either RsaI, RsaI plus HpaII or RsaI plus MspI followed by quantitative PCR. Per cent methylation was determined at each locus by comparing the quantity of amplified product after RsaI and HpaII digestion with the quantity of amplified product after cutting with RsaI alone. The RsaI and $M s p I$ digest was used to control for complete digestion. Retention of imprinting was defined as 30-70\% methylation of both ICR1 (H19DMR) and ICR2 (KvDMR); loss of imprinting as $80-100 \%$ methylation of ICR1 and $30-70 \%$ methylation of ICR2; LOH as $80-100 \%$ methylation of ICR1 and $0-20 \%$ methylation of ICR2.

Transfection analysis. The p117_118insNHL and the p.112_114PPV $>$ K mutations were generated using the QuikChange II Site-Directed Mutagenesis Kit (Agilent Technologies). The wild-type gene in a pCMV6-XL5 vector (OriGene) was used as the template. This cDNA clone is not tagged and therefore contains the native stop codon. The primer pairs used to generate the appropriate mutation were obtained by using the Agilent QuikChange Primer Design software program.

Primers used to generate the c.343 351dupAACCACCTG (p.117 118insNHL) mutant: sense: 5'-CCGTGAACCACCTGAACCACCTGCGCTGCGAGAAGCT-3'; antisense: 5'-AGCTTCTCGCAGCGCAGGTGGTTCAGGTGGTTCACGG-3'.

Primers used to generate the c.335_340delCGCCG (p.112_114PPV > L) mutant: Sense: 5'-CCTGGAAGGCAACCTGAACCACCTGCGC-3'; antisense: 5'-GCGCA GGTGGTTCAGGTTGCCTTCCAGG-3'.

The mutant strand synthesis reaction, the Dpn I digestion of the amplification products, and the transformation of the the XL10-Blue Gold ultracompetent cells were carried out according to the manufacturer's protocol. Briefly, 45 ul of XL10Gold ultracompetent cells were incubated with the $\beta$-ME mix, the Dpn I digestion product was added and incubated on ice for $30 \mathrm{~min}$, heat shocked at $42{ }^{\circ} \mathrm{C}$ for $30 \mathrm{~s}$, and incubated on ice for $2 \mathrm{~min}$. SOC broth was added and incubated at $37^{\circ} \mathrm{C}$ for $1 \mathrm{~h}$. Then, $100 \mathrm{ul}$ of the culture was spread on a pre-warmed culture plate containing ampicillin, and incubated overnight at $37^{\circ} \mathrm{C}$. The resulting colonies were transferred to a flask containing $5 \mathrm{ml}$ of LB media with ampicillin, and put on a shaker for $8 \mathrm{~h}$ at $37^{\circ} \mathrm{C}$. The plasmid was isolated from the cells using the Qiagen 
Plasmid Mini Kit, and was sequenced by using the sequencing primers provided with the Origene vector to verify the correct mutation.

HEK293 cells (purchased from American Type Culture Collection, Manassas, VA) were plated to achieve a confluency of 70-90\%. The cells were transfected with the pCMV6-XL5 vector without the gene insert (control), the pCMV6-XL5 vector with the wild-type gene, the pCMV6-XL5 vector with the mutant gene, or with a 1:1 ratio of the mutant and wild-type genes by using Lipofectamine 2000 according to the manufacturer's instructions. Three independent experiments were performed for each transfection. RNA was isolated from the cells 24 , and 48 and $72 \mathrm{~h}$ after transfection by using the Qiagen RNA Isolation Kit. Expression of the mutant was verified with the methodology described above for $M L L T 1$ gene expression analysis. The expression of selected genes of interest in the transfected HEK293 cells was performed using quantitative reverse transcription PCR using selected commercially available TaqMan Gene Expression Assay kits as described below.

Western analysis. The above transfected cells were lysed in RIPA buffer at 24, 48 and $72 \mathrm{~h}$ after transfection, and the lysates were separated using SDS-polyacrylamide gel electrophoresis. The proteins were transferred to polyvinylidene fluoride membranes, followed by blocking with $1 \%$ bovine serum albumin in PBS with $0.05 \%$ Tween, incubation for $1 \mathrm{~h}$ at room temperature with a MLLT1 antibody (1:1,000, diluted in blocking buffer; HPA031166, lot\#R30719, Sigma), and incubation for $30 \mathrm{~min}$ at room temperature with biotin-labelled secondary antibody (1:2,500 in blocking buffer; Rabbit AP, AP1000, lot\#Y0222, Vector Labs). Beta-actin (primary, 1:5,000, Anti-beta-actin, A5411, lot\#093K879, Sigma; secondary, 1:2,500, Mouse AP, AP2000, lot\#W0426, Vector Labs) was used for the loading control under the same conditions described for MLLT1. Bands were visualized by using the BCIP/NBT Kit (SK5400, Vector Labs). See Supplementary Fig. 2 for uncropped western blot.

Quantitative RT-PCR. Reverse transcription reactions, RNase inhibition and realtime PCR were performed according to the manufacturer's instructions for GAPDH, HOXA13 and HOTTIP. In brief, RNA was diluted to a concentration of $5 \mathrm{ng} \mu \mathrm{l}^{-1}$ and the reverse transcription reactions were performed using the High Capacity cDNA Reverse Transcription Kit (Life Technologies, cat\# 4368814) and RNase Inhibitor (Life Technologies, cat\# 100021540), per the manufacturer's instructions. Non-amplification controls were run once for each RNA sample. Real-time PCR was performed on $3 \mu \mathrm{l}$ of cDNA, according to the manufacturer's instructions, using the TaqMan Universal PCR Master Mix, No AmpErase UNG (Life Technologies, cat\# 4324018) and the 7500 Fast Real-Time PCR System. A non-template control was run with every plate, for each gene expression assay. The following Taqman gene expression assays were used: GAPDH (Life Technologies, cat\# Hs03929097_g1), HOXA13 (Life Technologies, cat\# Hs00426284_ml) and HOTTIP (Life Technologies, cat\# Hs00955374_s1). GAPDH was used as the endogenous control to calculate the dCt values for each sample. The ddCt value for the control sample at $24 \mathrm{~h}$ was used as the reference sample for calculating the relative quantitation (RQ) value in each sample for each gene $(R Q=2(-\mathrm{ddCt}))$. The data are shown as the average RQ and SEM for three independent experiments.

Computational protein structure analysis. The three-dimensional structure of residues 1-138 of wild-type MLLT1 YEATS was modelled using SwissModel ${ }^{62}$ based on the crystal structure of the $82 \%$ identical AF9 YEATS domain bound to H3K9ac (PDB id 4TMP). Mutant MLLT1 YEATS domains were produced using the same procedure. Models were visualized and analysed using Pymol (www.pymol.org).

Recombinant protein analysis. The codon-optimized DNA sequence coding for residues 1-138 of human MLLT1 (established using the Gibson assembly method) was inserted into the pET28 vector with thrombin cleavage site between 6 His tag and protein sequence. Proteins were expressed in Escherichia coli BL21(pLysS) cells. Expression was induced using $1 \mathrm{mM} \mathrm{IPTG}$, and allowed to proceed for $8 \mathrm{~h}$ at $20^{\circ} \mathrm{C}$. Wild-type and mutant proteins were purified using Ni-NTA sepharose followed by size exclusion chromatography (Pharmacia Superdex 75 16/600). Peak fractions were pooled. For ITC analysis proteins were dialyzed into $50 \mathrm{mM}$ Hepes buffer $\mathrm{pH} 7.4$, $300 \mathrm{mM} \mathrm{NaCl}, 5 \mathrm{mM} \beta$-mercaptoethanol and placed in the measurement cell (YEATS domain) or the injection syringe (histone peptides) of MicroCal ITC200 and TA Nano microcalorimeters, at $25^{\circ} \mathrm{C}$. Protein concentrations for wild-type, p.117_118insNHL, p.112_114PPV $>$ L and p.111_113NPP $>$ K mutants were 120, 130,120 and $90 \mu \mathrm{M}$, respectively. Peptide concentrations for all measurements were $1.2 \mathrm{mM}$. Thermal stability of wild-type and mutant proteins was probed using a Roche LightCycler 480II. Wild-type and mutant proteins were kept in the ITC buffer at concentrations of $7 \mu \mathrm{M}$. SyproOrange was used as dye, and temperature was ramped from $20-95^{\circ} \mathrm{C}$. Reported values are mean values of triplicate measurements.

Sequencing of selected genes. Probes to CTNNB1, WT1 and WTX were designed using RefSeq IDs limited to coding exons and UTRs with probe density specified at $2 \mathrm{x}$ with $98.7 \%$ of the target region covered. Pooled libraries were hybridized to the RNA probes. Post-capture material was purified and enriched with 10 cycles of PCR. Paired-end 100 base reads were sequenced per pool in a single lane of an Illumina HiSeq2500 instrument. Single nucleotide variants were filtered out if they were judged not to be damaging by at least $2 / 3$ of the following algorithms: SIFT using Ensembl Variant Effect Predictor Version 75 (ref. 63), PolyPhen Version 2 (ref. 10) or Provean Version 1.1.3 (ref. 64).

\section{References}

1. Rivera, M. N. \& Haber, D. A. Wilms tumour: connecting tumorigenesis and organ development in the kidney. Nat. Rev. Cancer 9, 699-712 (2005).

2. Beckwith, J. B., Kiviat, N. B. \& Bonadio, J. F. Nephrogenic rests, nephroblastomatosis, and the pathogenesis of Wilms Tumour. Pediatr. Pathol. 10, 1-36 (1990).

3. Beckwith, J. B. \& Palmer, N. F. Histopathology and prognosis of Wilms tumour. Results from the First National Wilms Tumour Study. Cancer 41, 1937-1948 (1978).

4. Bardeesy, N. et al. Anaplastic Wilms tumour, a subtype displaying poor prognosis, harbours p53 gene mutations. Nat. Genet. 7, 91-97 (1994).

5. Gadd, S. et al. Clinically relevant subsets identified by gene expression patterns support a revised ontogenic model of Wilms tumour: a Children's Oncology Group Study. Neoplasia 14, 742-756 (2012).

6. Walz, A. et al. Recurrent DGCR8, DROSHA, and SIX homeodomain mutations in favorable histology Wilms tumorus. Cancer Cell 27, 286-297 (2015).

7. Sherry, S. T. et al. dbSNP: the NCBI database of genetic variation. Nucleic Acids Res. 29, 308-311 (2001).

8. Forbes, S. A. et al. COSMIC: Mining complete cancer genomies in the catalogue of somatic mutations in Cancer. Nucleic Acids Res. 39, D945-D950 (2011).

9. McVean, G. A. et al. An integrated map of genetic variation from 1,092 human genomes. Nature 491, 56-65 (2012).

10. Adzhubei, I. A. et al. A method and server for predicting damaging missense mutations. Nat. Methods 7, 248-249 (2010).

11. Brunskill, E. W. \& Potter, S. S. RNA-Seq defines novel genes, RNA processing patterns and enhancer maps for the early stages of nephrogenesis: Hox supergenes. Dev. Biol. 368, 4-17 (2012).

12. Wang, K. C. et al. A long noncoding RNA maintains active chromatin to coordinate homeotic gene expression. Nature 472, 120-124 (2011).

13. Rinn, J. L. et al. Functional demarcation of active and silent chromatin domains in human HOX loci by noncoding RNAs. Cell 129, 1311-1323 (2007).

14. Olsson, P., Motegi, A., Bera, T. K., Lee, B. \& Pastan, I. PRAC2: a new gene expressed in human prostate and prostate cancer. Prostate 56, 123-130 (2003)

15. Ellis, B. C., Molloy, P. L. \& Graham, L. D. CRNDE: A Long Non-Coding RNA Involved in CanceR, Neurobiology, and DEvelopment. Front. Genet. 3, 270 (2012).

16. Grundy, P. E. et al. Loss of heterozygosity for chromosomes $1 \mathrm{p}$ and $16 \mathrm{q}$ is an adverse prognostic factor in favorable-histology Wilms tumour a report from the National Wilms Tumour Study Group. J. Clin. Oncol. 23, 7312-7321 (2005).

17. Gratias, E. J. et al. Gain of $1 \mathrm{q}$ is associated with inferior event-free and overall survival in patients with favorable histology Wilms tumour: a report from the Children's Oncology Group. Cancer 119, 3887-3894 (2013).

18. Schulze, J. M., Wang, A. Y. \& Kobor, M. S. YEATS domain proteins a diverse family with many links to chromatin modification and transcription. Biochem. Cell Biol. 87, 65-75 (2009).

19. Li, Y. et al. AF9 YEATS domain links histone acetylation to DOT1L-mediatied H3K79 Methylation. Cell 159, 558-571 (2014).

20. Benkert, P., Schwede, T. \& Tosatto, S. C. QMEANclust: estimation of protein model quality by combining a composite scoring function with structural density information. BMC Struct. Biol. 9, 35 (2009).

21. Lin, C. et al. AFF4, a component of the ELL/P-TEFb elongation complex and a shared subunit of MLL chimeras, can link transcription elongation to leukemia. Mol. Cell 37, 429-437 (2010).

22. Yokoyama, A., Lin, M., Naresh, A., Kitabayashi, I. \& Cleary, M. L. A higherorder complex containing AFF4 and ENL family proteins with P-TEFb facilitates oncogenic and physiologic MLL-dependent transcription. Cancer Cell 17, 198-212 (2010).

23. He, N. et al. Human Polymerase-Associated Factor complex (PAFc) connects the Super Elongation Complex (SEC) to RNA polymerase II on chromatin. Proc. Natl Acad. Sci. USA 108, E636-E645 (2011).

24. Luo, Z., Lin, C. \& Shilatifard, A. The super elongation complex (SEC) family in transcriptional control. Nat. Rev. Mol. Cell Biol. 13, 543-547 (2012).

25. Peterlin, B. M. \& Price, D. H. Controlling the elongation phase of transcription with P-TEFb. Mol. Cell 23, 297-305 (2006).

26. Doty, R. T., Vanasse, G. J., Disteche, C. M. \& Willerford, D. M. The leukemiaassociated gene Mllt1/ENL: characterization of a murine homolog and demonstration of an essential role in embryonic development. Blood Cells $\mathrm{Mol}$. Dis. 28, 407-417 (2002).

27. Krivtsov, A. V. \& Armstrong, S. A. MLL translocations, histone modifications and leukaemia stem-cell development. Nat. Rev. Cancer 11, 823-833 (2007).

28. Slany, R. K. The molecular biology of mixed lineage leukemia. Haematologica 94, 984-993 (2009)

29. Mueller, D. et al. Misguided transcriptional elongation causes mixed lineage leukemia. PLoS Biol. 7, e1000249 (2009).

30. Zeisig, D. T. et al. The eleven-nineteen-leukemia protein ENL connects nuclear MLL fusion partners with chromatin. Oncogene 24, 5525-5532 (2005). 
31. Schulze, J. M., Wang, A. Y. \& Kobor, M. S. Reading chromatin: insights from yeast into YEATS domain structure and function. Epigenetics 5, 573-577 (2010).

32. Mueller, D. et al. A role for the MLL fusion partner ENL in transcriptional elongation and chromatin modification. Blood 110, 4445-4454 (2007).

33. Bitoun, E., Oliver, P. L. \& Davies, K. E. The mixed-lineage leukemia fusion partner AFF4 stimulates RNA polymerase II transcriptional elongation and mediates coordinated chromatin remodeling. Hum. Mol. Genet. 16, 92-106 (2007).

34. Zhang, W., Xia, X., Reisenauer, M. R., Hemenway, C. S. \& Kone, B. C. DotlaAF9 complex mediates histone $\mathrm{H} 3$ Lys-79 hypermethylation and repression of ENaCalpha in an aldosterone-sensitive manner. J. Biol. Chem. 281, 18059-18068 (2006)

35. Jones, B. et al. The histone H3K79 methyltransferase Dot1L is essential for mammalian development and heterochromatin structure. PLoS Genet. 12, e1000190 (2008).

36. Steger, D. J. et al. DOT1L/KMT4 recruitment and H3K79 methylation are ubiquitously coupled with gene transcription in mammalian cells. Mol. Cell Biol. 28, 2825-2839 (2008).

37. Chaudhary, K., Deb, S., Moniaux, N., Ponnusamy, M. P. \& Batra, S. K. Human RNA polymerase II-associated factor complex: dysregulation in cancer. Oncogene 26, 7499-7507 (2007)

38. Kim, J. \& Roeder, R. G. Direct Bre1-Paf1 complex interactions and RING finger-independent Bre1-Rad6 interactions mediate histone H2B ubiquitylation in yeast. J. Biol. Chem. 284, 20582-20592 (2009).

39. Chopra, V. S., Hong, J. W. \& Levine, M. Regulation of Hox gene activity by transcriptional elongation in Drosophila. Curr. Biol. 19, 688-693 (2009).

40. Lin, C. et al. Dynamic transcriptional events in embryonic stem cells mediated by the super elongation complex (SEC). Genes Dev. 25, 1486-1498 (2011).

41. Luo, Z. et al. The super elongation complex family of RNA polymerase II elongation factors gene target specificity and transcriptional output. Mol. Cell. Biol. 32, 2608-2617 (2012).

42. Hanks, S. et al. Germline mutations in the PAF1 complex gene CTR9 predispose to Wilms tumour. Nat. Commun. 5, 4398 (2014).

43. Yoo, H.-S., Seo, J.-H. \& Yoo, J.-Y. CTR9, a component of the PAF complex, controls elongation block at the $\mathrm{c}$-fos locus via signal-dependent regulation of chromatin bound NELF dissociation. PLoS One 8, e61055 (2013).

44. Carpten, J. D. et al. HRPT2, encoding parafibromin, is mutated in hyperparathyroidism-jaw tumour syndrome. Nat. Genet. 32, 676-680 (2002).

45. Scott, R. H., Stiller, C. A., Wlaker, L. \& Rhaman, N. Syndromes and constitutional chromosomal abnormalities associated with Wilms tumour. J. Med. Genet. 43, 705-715 (2006).

46. Heppe, R. K., Koyle, M. A. \& Beckwith, J. B. Nephrogenic rests in Wilms tumour patients with the Drash syndrome. J. Urol. 145, 1225-1228 (1991).

47. Maiti, S., Alam, R., Amos, C. I. \& Huff, V. Frequent Association of B-Catenin and WT1 Mutations in Wilms Tumours. Cancer Res. 60, 6288-6292 (2000).

48. Basu, M. \& Roy, S. S. Wnt/ $\beta$-catenin pathway is regulated by PITX2 homeodomain protein and thus contributes to the proliferation of human ovarian adenocarcinoma cell, SKOV-3. J. Biol. Chem. 288, 4355-4367 (2013).

49. Kioussi, C. et al. Identification of a Wnt/Dvl/beta-Catenin --> Pitx2 pathway mediating cell-type-specific proliferation during development. Cell 111, 673-685 (2002).

50. Han, Y. et al. Identification and validation that up-expression of HOXA13 is a novel independent prognostic marker of a worse outcome in gastric cancer based on immunohistochemistry. Med. Oncol. 30, 564 (2013).

51. Guttman, M. et al. LincRNAs act in the circuitry controlling pluripotency and differentiation. Nature 477, 295-300 (2011).

52. Prentice, R. L. A case-cohort design for epidemiologic cohort studies and disease prevention trials. Biometrika 73, 1-11 (1986).

53. Malogolowkin, M. et al. Incidence and outcomes of patients with late recurrence of Wilms Tumour. Pediatr. Blood Cancer 60, 1612-1615 (2013).

54. Drmanac, R. et al. Human genome sequencing using unchained base reads on self-assembling DNA nanoarrays. Science 327, 78-81 (2010).

55. Bainbridge, M. N. et al. De novo truncating mutations in ASXL3 are associated with a novel clinical phenotype with similarities to Bohring-Opitz syndrome. Genome Med. 5, 11 (2013).

56. Lupski, J. R. et al. Exome sequencing resolves apparent incidental findings and reveals further complexity of SH3TC2 variant alleles causing CMT neuropathy. Genome Med. 5, 57-70 (2013).

57. Edmonson et al. Bambino: a variant detector and alignment viewer for next-generation sequencing data in the SAM/BAM format. Bioinformatics 27, 865-866 (2011).

58. Pounds, S. et al. Reference alignment of SNP microarray signals for copy number analysis of tumours. Bioinformatics 25, 315-321 (2009).

59. Brunet, J. P., Tamayo, P., Golub, T. R. \& Mesirov, J. P. Metagenes and molecular pattern discovery using matrix factorization. Proc. Natl Acad. Sci. USA 101, 4164-4169 (2004).

60. Tusher, V., Tibshirani, R. \& Chu, G. Significance analysis of microarrays applied to transcriptionalresponses to ionizing radiation. Proc. Natl Acad. Sci. USA 98, 5116-5121 (2001).
61. Perlman, E. J. et al. WT1 mutation and 11P15 loss of heterozygosity predict relapse in very low-risk Wilms tumorus treated with surgery alone: a Children's Oncology Group Study. J. Clin. Oncol. 29, 698-703 (2011).

62. Arnold, K., Bordoli, L., Kopp, J. \& Schwede, T. The SWISS-MODEL workspace: a web-based environment for protein structure homology modelling. Bioinformatics 22, 195-201 (2006).

63. McLaren, W. et al. Deriving the consequences of genomic variants with Ensembl API and SNP Effect Predictor. Bioinformatics 26, 2069-2070 (2010).

64. Choi, Y., Sims, G. E., Murphy, S., Miller, J. R. \& Chan, A. P. Predicting the functional effect of amino acid substitutions and indels. PLoS ONE 7, e46688 (2012).

\section{Acknowledgements}

The TARGET initiative is supported by NCI Grant U10 CA98543. Work performed under contracts from the National Cancer Institute, US National Institutes of Health within HHSN261200800001E include specimen processing (the Children's Oncology Group Biopathology Center (BPC), whole genomic sequencing (Complete Genomics, Inc), whole exomic sequencing (Baylor College of Medicine) and specific capture followed by sequencing (British Columbia Cancer Agency Genome Sciences Centre). The content of this publication does not necessarily reflect the views of policies of the Department of Health and Human Services, nor does mention of trade names, commercial products, or organizations imply endorsement by the US Government. The authors thank Patee Gesuwan and Leandro Hermida and the Data Coordinating Center for their support. We thank Karen Carty and Franceline Huser and for help and assistance with the recombinant protein production and DSF analysis. We thank the KAUST bioscience core lab for access to the MicroCal ITC200 microcalorimeter. We also thank the Clinical Applications of Core Technology Laboratory of the Hartwell Center for Bioinformatics and Biotechnology of St Jude Children's Research Hospital for performing the copy number analysis. The authors are grateful for the project management expertize of Karen Novik and Laura Monovich, and for the technical expertize of Patricia Beezhold, Donna Kersey, Debbie-Payne Turner, Mary McNulty and Yvonne Moyer. This work would not be possible without the dedication of all the experts within the many disciplines both at the local institutions and centrally within the Children's Oncology Group. They enable the coordination and accurate annotation required for the therapeutic and biology protocols that form the basis for the TARGET studies. Financial Support: NCI U10 CA98543 (E.J.P., J.M.G.-F. and M.A.M.); NIH U10CA42326 (E.J.P.); U10CA98543 (J.S.D. and E.J.P.); U24 CA114766; UO1CA88131 (E.J.P.), the IDP Foundation (E.J.P.), the American and Lebanese Syrian Associated Charities of St Jude (J.M. and C.M.) and the King Abdullah University of Science and Technology (S.T.A. and A.R.).

\section{Author contributions}

D.S.G., J.M.G.A., M.A.S. and T.M.D. oversaw the administrative and data management aspects of the TARGET project; L.J. performed the MLLT1 fragment analysis; D.M., C.H.H., C.N. provided the bioinformatic analysis; S.T.A. and A.R. provided the in silico protein structure analysis and the biophysical analysis of recombinant protein; J.A. provided the statistical analysis; J.M.G.-F. and N.R. performed the specimen processing and quality control; J.S.D. and V.H. provided the samples and the clinical data; C.G.M. and J.M. performed the copy number analysis; S.G. performed the gene expression analysis; Y.M., A.J.M., R.A.M. and M.A.M. performed the target capture sequencing; E.J.P. designed and oversaw all aspects of the study and wrote the paper with contributions from all other authors.

\section{Additional information}

Accession codes: Whole genome and whole exome sequencing data have been deposited in the dbGAP database under the accession code phs000471.

Supplementary Information accompanies this paper at http://www.nature.com/ naturecommunications

Competing financial interests: The authors declare no competing financial interests.

Reprints and permission information is available online at http://npg.nature.com/ reprintsandpermissions/

How to cite this article: Perlman, E. J. et al. MLLT1 YEATS domain mutations in clinically distinctive Favourable Histology wilms tumours. Nat. Commun. 6:10013 doi: 10.1038/ncomms10013 (2015)

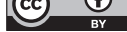

This work is licensed under a Creative Commons Attribution 4.0 International License. The images or other third party material in this article are included in the article's Creative Commons license, unless indicated otherwise in the credit line; if the material is not included under the Creative Commons license, users will need to obtain permission from the license holder to reproduce the material. To view a copy of this license, visit http://creativecommons.org/licenses/by/4.0/ 